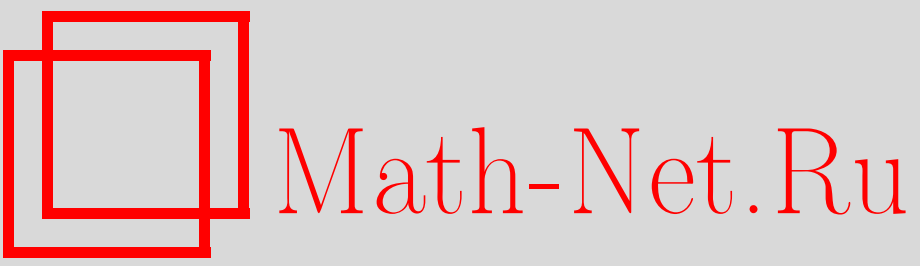

Я. Антонио, Г. П. Пронько, Гамильтонов формализм в гидродинамике, ТМФ, 2004, том 141, номер 3, 392-410

DOI: https://doi.org/10.4213/tmf127

Использование Общероссийского математического портала Math-Net.Ru подразумевает, что вы прочитали и согласны с пользовательским соглашением

http://www.mathnet.ru/rus/agreement

Параметры загрузки:

IP: 54.224 .135 .184

26 апреля 2023 г., 04:12:26 


\author{
ТЕОРЕТИЧЕСКАЯ \\ И МАТЕМАТИЧЕСКАЯ \\ ФИЗИКА \\ Том 141, № 3 \\ декабрь, 2004
}

(C) 2004 г. Я. Антонио*, Г.П. Пронько*, $\dagger$

\title{
ГАМИЛЬТОНОВ ФОРМАЛИЗМ В ГИДРОДИНАМИКЕ
}

Для описания динамики жидкости предложен гамильтонов подход, основанный на формулируемой в терминах лагранжевых переменных динамике. Построение канонических переменных жидкости проливает свет на происхождение введенных в девятнадцатом веке переменных Клебша. Развитый формализм позволяет связать закон сохранения циркуляции (теорему Томсона) с инвариантностью теории относительно специальных диффеоморфизмов, а также установить новые законы сохранения. Обсуждается различие между эйлеровым и лагранжевым описаниями, при этом указывается на неполноту первого. Построенный формализм применим также к идеальной плазме. В заключение приведены некоторые замечания относительно квантования жидкости.

Ключевые слова: гидродинамика, канонические переменные, плазма.

\section{1. ВВЕДЕНИЕ}

В настоящее время почти все фундаментальные физические явления могут быть рассмотрены в рамках как классической, так и квантовой механики. Это означает, что такие явления допускают гамильтоново описание. За свою долгую историю гамильтонов формализм дал много мощных методов анализа общих свойств эволюции систем, инструментов для решения частных задач, а также уникальный способ перехода от классического описания систем к квантовому описанию.

В этом отношении механика сплошных сред (гидродинамика), несмотря на свое название, стоит в стороне от традиционной механики. Причиной этого является не только бесконечное число степеней свободы жидкости. Мы уже научились формулировать теорию классических и квантовых полей. Основное различие меж ду обычной теорией поля и теорией сплошных сред заключается в том, что в первом случае речь может идти о динамике поля в фиксированной точке пространства (соответствующее поле, разумеется, взаимодействует с полем в соседних точках), в то время как во втором случае при описании взаимодействия составляюших жидкость соседних частиц положение частишы в пространстве не фиксировано вследствие движения жидкости. В то же время задачей

\footnotetext{
${ }^{*}$ Chaos and Innovation Research Unit, Department of Mathematics, Aristoteles University of Thesaloniki, Greece. E-mail: iantonio@math.auth.gr

${ }^{\dagger}$ Институт физики высоких энергий, Протвино, Московская обл., Россия. E-mail: pronko@mx.ihep.su
} 
обычной гидродинамики является определение скорости, плотности и значения термодинамической переменной (давления или энтропии) как функций от координат $\vec{x}$ и времени $t$ для соответствуюших граничных и/или начальных условий [1]. Аналогичные задачи возникают также в магнитогидродинамике достаточно плотной плазмы [2]. Для развития гамильтонова формализма необходимо начать с более детального описания, основанного на траекториях частиц, составляюших жидкость или плазму. Такое описание особенно важно в случае плазмы, поскольку фундаментальное электромагнитное взаимодействие может быть определено только в терминах траекторий зарядов. Необходимо отметить, что некоторые аспекты такого подхода подробно рассматривались в ряде работ Марсдена, Вейнстейна, Купершмидта, Нолма, Ратиу и Левермора [3] особенно в контексте проблемы устойчивости для идеальной жидкости. В отличие от этих работ, мы сознательно избегаем, где это возможно, использования языка современной дифференциальной геометрии, чтобы сделать нашу статью понятной для физиков.

Конечно, не все свойства жидкости могут быть учтены в рамках гамильтонова подхода. Например, мы оставляем открытыми вопросы, связанные с диссипацией энергии, вязкостью и т.д.

\section{2. ЛАГРАНЖЕВЫ УРАВНЕНИЯ ДВИЖЕНИЯ}

В гидродинамике приняты две различные картины описания. Первая, обычно называемая эйлеровой, в качестве координат использует пространственно зависимые поля скорости, плотности и некоторую термодинамическую переменную. Вторая, лагранжева, использует координаты частиц $\vec{x}\left(\xi_{i}, t\right)$, отмеченные набором параметров $\xi_{i}$, которые можно рассматривать как начальные положения $\vec{\xi}=\left.\vec{x}(t)\right|_{t=0}$, и временем $t$. Эти начальные положения $\vec{\xi}$, так же как и координаты $\vec{x}\left(\xi_{i}, t\right)$, принадлежат некоторой области $D \subseteq \mathbb{R}^{3}$. Ниже мы будем рассматривать только консервативные системы, в которых траектории различных частиц не пересекаются, поэтому ясно, что функции $\vec{x}\left(\xi_{i}, t\right)$ определяют диффеоморфизм области $D \subseteq \mathbb{R}^{3}$ и что функции, обратные $\vec{\xi}\left(x_{i}, t\right)$, также должны сушествовать:

$$
\begin{aligned}
\left.x_{j}\left(\xi_{i}, t\right)\right|_{\vec{\xi}=\vec{\xi}\left(x_{i}, t\right)} & =x_{j}, \\
\left.\xi_{j}\left(x_{i}, t\right)\right|_{\vec{x}=\vec{x}\left(\xi_{i}, t\right)} & =\xi_{j} .
\end{aligned}
$$

Плотность частиц в пространстве в момент времени $t$ есть

$$
\rho(\vec{x}, t)=\int d^{3} \xi \rho_{0}\left(\xi_{i}\right) \delta\left(\vec{x}-\vec{x}\left(\xi_{i}, t\right)\right),
$$

где $\rho_{0}(\xi)$ - начальная плотность в момент времени $t=0$. Поле скорости $\vec{v}$ как функция от координат $\vec{x}$ и $t$ есть

$$
\vec{v}\left(x_{i}, t\right)=\dot{\vec{x}}\left(\vec{\xi}\left(x_{i}, t\right), t\right),
$$

где $\vec{\xi}(x, t)$ - обратная функция (1). Скорость может быть также записана в следуюшем виде:

$$
\vec{v}\left(x_{i}, t\right)=\frac{\int d^{3} \xi \rho_{0}\left(\xi_{i}\right) \dot{\vec{x}}\left(\xi_{i}, t\right) \delta\left(\vec{x}-\vec{x}\left(\xi_{i}, t\right)\right)}{\int d^{3} \xi \rho_{0}\left(\xi_{i}\right) \delta\left(\vec{x}-\vec{x}\left(\xi_{i}, t\right)\right)},
$$


или

$$
\rho\left(x_{i}, t\right) \vec{v}\left(x_{i}, t\right)=\int d^{3} \xi \rho_{0}\left(\xi_{i}\right) \dot{\vec{x}}\left(\xi_{i}, t\right) \delta\left(\vec{x}-\vec{x}\left(\xi_{i}, t\right)\right) .
$$

Вычислим производную по времени от плотности, используя определение последней в виде (2):

$$
\begin{aligned}
\dot{\rho}\left(x_{i}, t\right) & =\int d^{3} \xi \rho_{0}\left(\xi_{i}\right) \frac{\partial}{\partial t} \delta\left(\vec{x}-\vec{x}\left(\xi_{i}, t\right)\right)= \\
& =\int d^{3} \xi \rho_{0}\left(\xi_{i}\right)\left(-\dot{\vec{x}}\left(\xi_{i}, t\right)\right) \frac{\partial}{\partial \vec{x}} \delta\left(\vec{x}-\vec{x}\left(\xi_{i}, t\right)\right)= \\
& =-\frac{\partial}{\partial \vec{x}} \int d^{3} \xi \rho_{0}\left(\xi_{i}\right) \dot{\vec{x}}\left(\xi_{i}, t\right) \delta\left(\vec{x}-\vec{x}\left(\xi_{i}, t\right)\right)=-\frac{\partial}{\partial \vec{x}} \rho\left(x_{i}, t\right) \vec{v}\left(x_{i}, t\right) .
\end{aligned}
$$

Таким образом мы убеждаемся в справедливости уравнения непрерывности для гидродинамики:

$$
\dot{\rho}\left(x_{i}, t\right)+\vec{\partial}\left(\rho\left(x_{i}, t\right) \vec{v}\left(x_{i}, t\right)\right)=0 .
$$

Используя координаты $\vec{x}\left(\xi_{i}, t\right)$ как переменные в конфигурационном пространстве, можно рассмотреть простейшее движение жидкости, описываемое лагранжианом

$$
L=\int d^{3} \xi \frac{m \dot{\vec{x}}^{2}\left(\xi_{i}, t\right)}{2}
$$

Вытекающие из (8) уравнения движения, очевидно, имеют вид

$$
m \ddot{\vec{x}}\left(\xi_{i}, t\right)=0 \text {. }
$$

Установим теперь, что это уравнение означает для плотности и скорости жидкости. Для этого продифференцируем обе части выражения (5) по времени:

$$
\begin{aligned}
\frac{\partial}{\partial t} \rho\left(x_{i}, t\right) \vec{v}\left(x_{i}, t\right)= & \int d^{3} \xi \rho_{0}\left(\xi_{i}\right) \ddot{\vec{x}}\left(\xi_{i}, t\right) \delta\left(\vec{x}-\vec{x}\left(\xi_{i}, t\right)\right)+ \\
& +\int d^{3} \xi \rho_{0}\left(\xi_{i}\right) \dot{\vec{x}}\left(\xi_{i}, t\right) \frac{\partial}{\partial t} \delta\left(\vec{x}-\vec{x}\left(\xi_{i}, t\right)\right) .
\end{aligned}
$$

Первый член в правой части этого равенства обрашается в нуль в силу уравнений движения (9), и, преобразуя второй член так, как это было сделано в (6), мы приходим к равенству

$$
\frac{\partial}{\partial t} \rho\left(x_{i}, t\right) \vec{v}\left(x_{i}, t\right)+\frac{\partial}{\partial x_{k}}\left(\rho\left(x_{i}, t\right) \vec{v}\left(x_{i}, t\right) v_{k}\left(x_{i}, t\right)\right)=0 .
$$

Перепишем его в следуюшем виде:

$$
\vec{v}\left(x_{i}, t\right)\left[\dot{\rho}\left(x_{i}, t\right)+\frac{\partial}{\partial x_{k}}\left(\rho\left(x_{i}, t\right) v_{k}\left(x_{i}, t\right)\right)\right]+\rho\left(x_{i}, t\right)\left[\dot{\vec{v}}\left(x_{i}, t\right)+v_{k}\left(x_{i}, t\right) \frac{\partial}{\partial x_{k}} \vec{v}\left(x_{i}, t\right)\right]=0 .
$$


Первый член здесь обрашается в нуль в силу уравнения непрерывности, тогда как второй дает уравнение Эйлера в случае свободного потока:

$$
\dot{\vec{v}}\left(x_{i}, t\right)+v_{k}\left(x_{i}, t\right) \frac{\partial}{\partial x_{k}} \vec{v}\left(x_{i}, t\right)=0 .
$$

Чтобы двигаться дальше, нам необходимо ввести в лагранжиан (8) отвечающий “потенциальной энергии" член, который приведет к появлению поля внутреннего давления в уравнении Эйлера. Как было отмечено выше, функции $\vec{x}\left(\xi_{i}, t\right)$ определяют диффеоморфизм в $\mathbb{R}^{3}$, поэтому матрица Якоби

$$
A_{k}^{j}\left(\xi_{i}, t\right)=\frac{\partial x_{j}\left(\xi_{i}, t\right)}{\partial \xi_{k}}
$$

является невырожденной для любых $\xi_{i}$ и $t$. Интеграл (2) может быть выражен через детерминант матрицы Якоби $A_{k}^{j}\left(\xi_{i}, t\right)$ :

$$
\rho(\vec{x}, t)=\left.\frac{\rho_{0}\left(\xi_{i}\right)}{\operatorname{det} A\left(\xi_{i}, t\right)} g\right|_{\vec{\xi}=\vec{\xi}\left(x_{i}, t\right)} .
$$

Предположим для простоты, что начальная плотность $\rho_{0}\left(\xi_{i}\right)$ постоянна в области $D \subseteq \mathbb{R}^{3}$, и эффективно нормируем поле плотности $\rho\left(\xi_{i}, t\right)$, положив $\rho_{0}\left(\xi_{i}\right)=1$ (одна частица в элементарном объеме). Тогда равенство (15) принимает вид

$$
\rho(\vec{x}, t)=\left.\frac{1}{\operatorname{det} A\left(\xi_{i}, t\right)}\right|_{\vec{\xi}=\vec{u}\left(x_{i}, t\right)} .
$$

Рассмотрим в качестве “потенциальной энергии” функционал от якобиана $\operatorname{det} A$ :

$$
L=\int d^{3} \xi\left[\frac{m \dot{\vec{x}}^{2}\left(\xi_{i}, t\right)}{2}-f\left(\operatorname{det} A\left(\xi_{i}, t\right)\right)\right] .
$$

Теперь уравнения движения принимают вид

$$
m \ddot{x}_{j}\left(\xi_{i}, t\right)-\frac{\partial}{\partial \xi_{k}}\left(\left(A^{-1}\right)_{j}^{k}\left(\xi_{i}, t\right) f^{\prime}(\operatorname{det} A) \operatorname{det} A\right)=0 .
$$

Подставляя $\ddot{x}_{j}\left(\xi_{i}, t\right)$ из $(18)$ в уравнение $(10)$ и действуя так же, как при выводе уравнения (13), получаем

$$
m \rho\left(x_{i}, t\right)\left(\frac{\partial}{\partial t}+v_{k}\left(x_{i}, t\right) \frac{\partial}{\partial x_{k}}\right) v_{j}\left(x_{i}, t\right)-\frac{\partial}{\partial x_{j}}\left(\left.f^{\prime}\left(\operatorname{det} A\left(\xi_{i}, t\right)\right)\right|_{\vec{\xi}=\vec{\xi}\left(x_{i}, t\right)}\right)=0 .
$$

Теперь очевидно, что если отождествить $-\left.(1 / m) f^{\prime}\left(\operatorname{det} A\left(\xi_{i}, t\right)\right)\right|_{\vec{\xi}=\vec{\xi}\left(x_{i}, t\right)}$ с давлением $p\left(x_{i}, t\right)$, то уравнение (19) примет вид обычного уравнения Эйлера в отсутствие вязкости:

$$
\rho\left(x_{i}, t\right)\left(\frac{\partial}{\partial t}+v_{k}\left(x_{i}, t\right) \frac{\partial}{\partial x_{k}}\right) v_{j}\left(x_{i}, t\right)=-\frac{\partial}{\partial x_{j}} p\left(x_{i}, t\right) .
$$

Отметим, что появившееся здесь давление $p\left(x_{i}, t\right)$ связано не с наличием внешней силы, а является результатом взаимодействия между частицами. Дальнейшее усложнение лагранжиана в настоящий момент не является необходимым. Одно замечание, тем не менее, должно быть сделано. Никакое изменение лагранжиана не позволит нам получить уравнение Навье-Стокса, поскольку последнее учитьвает эффекты диссипации, которые не симметричны по времени. 


\section{3. ГАМИЛЬТОНОВ ФОРМАЛИЗМ}

Гамильтонов формализм классической механики наряду с другими преимушествами дает нам уникальньй способ построения квантовой теории. В этом отношении гидродинамика стоит в стороне, поскольку ее основные переменные - локальные скорость, плотность и термодинамическая функция - не допускают непосредственной гамильтоновой интерпретации, несмотря на то что довольно часто в учебниках можно встретить термины типа "плотность импульса" (см., например, [1]).

Мы введем канонические переменные, соответствующие лагранжиану (17), таким образом, каноническими координатами у нас будут функции $\vec{x}\left(\xi_{i}, t\right)$. Сопряженные к ним импульсы определяются как производные от лагранжиана по скоростям $\dot{\vec{x}}\left(\xi_{i}, t\right)$ :

$$
\vec{p}\left(\xi_{i}, t\right)=\frac{\delta L}{\delta \dot{\vec{x}}\left(\xi_{i}, t\right)}=m \dot{\vec{x}}\left(\xi_{i}, t\right) .
$$

Гамильтониан дается преобразованием Лежандра от лагранжиана:

$$
H=\int d^{3} \xi\left(\frac{1}{2 m} \vec{p}^{2}\left(\xi_{i}, t\right)+f\left(\operatorname{det} A\left(\xi_{i}, t\right)\right)\right) .
$$

Канонические скобки Пуассона определены следующим образом:

$$
\left\{p_{j}\left(\xi_{i}\right), x_{k}\left(\xi_{i}^{\prime}\right)\right\}=\delta_{j k} \delta^{3}\left(\xi_{i}-\xi_{i}^{\prime}\right)
$$

Очевидно, скобки Пуассона (23) и гамильтониан (22) определяют уравнения движения в канонических переменных, эквивалентные уравнениям Лагранжа. Фазовое пространство Г жидкости образовано переменными $\vec{x}\left(\xi_{i}, t\right), \vec{p}\left(\xi_{i}, t\right)$. В настоящий момент у нас отсутствует пространственная интерпретация переменных $\vec{x}\left(\xi_{i}\right)$ и $\vec{p}\left(\xi_{i}\right)$. Чтобы получить такую интерпретацию, введем новый объект, используя то же усреднение, что было использовано в предыдушем разделе:

$$
\vec{l}(x)=\int d^{3} \xi \vec{p}\left(\xi_{i}\right) \delta\left(\vec{x}-\vec{x}\left(\xi_{i}\right)\right)=\rho(x) \vec{p}\left(\xi_{i}(x)\right) .
$$

Этот новый объект будет играть роль части координат в фазовом пространстве Г, следовательно, нам нужно вычислить его скобки Пуассона, используя (23). Результат имеет следующий вид:

$$
\left\{l_{j}\left(x_{i}\right), l_{k}\left(y_{i}\right)\right\}=\left[l_{k}\left(x_{i}\right) \frac{\partial}{\partial x_{j}}+l_{j}\left(y_{i}\right) \frac{\partial}{\partial x_{k}}\right] \delta(\vec{x}-\vec{y}) .
$$

Такие скобки Пуассона были давно введены из геометрических соображений без физического обоснования в работах [4] как “скобки гидродинамического типа". Настоящее обсуждение обнаруживает происхождение этих скобок. Коммутационное соотношение $(25)$ совпадает с алгеброй трехмерных диффеоморфизмов, где $l_{j}\left(x_{i}\right)$ служит в 
качестве генераторов. Другими словами, с помощью этих генераторов можно реализовать конечный диффеоморфизм $x_{j} \rightarrow \phi_{j}\left(x_{i}\right)$ любой $x$-зависимой динамической переменной. Следует отметить, что порождаемая генераторами $l_{j}\left(x_{i}\right)$ группа диффеоморфизмов в случае гидродинамики не является калибровочно-симметричной, каковой она является, например, в случае релятивистской струны или мембраны. В то же время в гидродинамике сушествует бесконечномерная симметрия по отношению к специальным (т.е. сохраняюшим объем) диффеоморфизмам SDiff, которые будут рассмотрены в следуюшем разделе.

Как было упомянуто выше, величины $l_{j}\left(x_{i}\right)$ являются только частью $x$-зависимых координат в фазовом пространстве $\Gamma$; они могут рассматриваться как " $x$-зависимые импульсы”. Теперь мы должны определить “х-зависимые координаты”. На эту роль можно выбрать, например, функции $\xi_{j}\left(x_{i}\right)$, которые являются обратными к функциям $x_{j}\left(\xi_{i}\right)(1)$. Дифференцируя первое из равенств (1) по $x$, получаем

$$
A_{k}^{j}\left(\xi_{i}\left(x_{k}\right)\right) \frac{\partial \xi_{k}\left(x_{i}\right)}{\partial x_{l}}=\delta_{l}^{j}
$$

другими словами, матрища

$$
a_{l}^{k}\left(x_{i}\right)=\frac{\partial \xi_{k}\left(x_{i}\right)}{\partial x_{l}}
$$

является обратной к матрице $A_{k}^{j}\left(\xi_{i}\left(x_{k}\right)\right)$. Поэтому из (16) следует, что

$$
\rho\left(x_{i}\right)=\operatorname{det} a\left(x_{i}\right) .
$$

Чтобы упростить вычисление скобок Пуассона, можно выразить $\xi_{j}\left(x_{i}\right)$ следующим образом:

$$
\xi_{j}\left(x_{i}\right)=\frac{\int d^{3} \xi \xi_{j} \delta\left(\vec{x}-\vec{x}\left(\xi_{i}, t\right)\right)}{\int d^{3} \xi \delta\left(\vec{x}-\vec{x}\left(\xi_{i}, t\right)\right)} .
$$

Отсюда легко получаем

$$
\left\{\xi_{j}\left(x_{i}\right), \xi_{k}\left(y_{i}\right)\right\}=0 .
$$

Вычисление скобок Пуассона между величинами $\xi_{j}\left(x_{i}\right)$ и $l_{j}\left(x_{i}\right)$ более сложно, но результат прост:

$$
\left\{l_{j}\left(x_{i}\right), \xi_{k}\left(y_{i}\right)\right\}=-\frac{\partial \xi_{k}\left(x_{i}\right)}{\partial x_{j}} \delta(\vec{x}-\vec{y}) .
$$

Таким способом мы построили набор $x$-зависимых координат $\left(l_{j}\left(x_{i}\right), \xi_{j}\left(x_{i}\right)\right)$ в фазовом пространстве Г, однако преобразование

$$
\left(p_{j}\left(\xi_{i}\right), x_{j}\left(\xi_{i}\right)\right) \rightarrow\left(l_{j}\left(x_{i}\right), \xi_{j}\left(x_{i}\right)\right)
$$

не является каноническим. Набор канонических $x$-зависимых координат в фазовом пространстве Г может быть получен следующим образом. Умножим обе части равенства (31) на матрицу $A_{m}^{j}\left(\xi_{i}\left(x_{k}\right)\right)$ :

$$
A_{m}^{j}\left(\xi_{i}\left(x_{k}\right)\right)\left\{l_{j}\left(x_{i}\right), \xi_{k}\left(y_{i}\right)\right\}=-\delta_{m}^{k} \delta(\vec{x}-\vec{y}),
$$


где использовано равенство (26). Далее, в соответствии с соотношением (30) можно внести $A_{m}^{j}\left(\xi_{i}\left(x_{k}\right)\right)$ внутрь скобок и получить

$$
\left\{\pi_{m}\left(x_{i}\right), \xi_{k}\left(y_{i}\right)\right\}=\delta_{m}^{k} \delta(\vec{x}-\vec{y}),
$$

где

$$
\pi_{m}\left(x_{i}\right)=-A_{m}^{j}\left(\xi_{i}\left(x_{k}\right)\right) l_{j}\left(x_{i}\right) .
$$

Прямым вычислением получим также

$$
\left\{\pi_{m}\left(x_{i}\right), \pi_{k}\left(y_{i}\right)\right\}=0,
$$

так что набор $\left(\pi_{m}\left(x_{i}\right), \xi_{k}\left(y_{i}\right)\right)$ образован каноническими переменными. В терминах этих канонических переменных генераторы группы диффеоморфизмов $l_{j}\left(x_{i}\right)$ имеют следуюший вид:

$$
l_{j}\left(x_{i}\right)=-\frac{\partial \xi_{k}\left(x_{i}\right)}{\partial x_{j}} \pi_{k}\left(x_{i}\right) .
$$

Для рассматриваемого лагранжиана из $(21)$ и $(24)$ следует, что генераторы $l_{j}\left(x_{i}\right)$ даются формулой

$$
l_{j}\left(x_{i}\right)=m \rho\left(x_{i}\right) v_{j}\left(x_{i}\right),
$$

и представление (37) очень похоже на параметризацию Клебша [5] для скорости. Отличие (37) от первоначальной параметризации Клебша состоит в появлении трех "потенциалов" вместо двух для трехмерной жидкости. Причина такого различия будет обсуждаться в конще настоящего раздела.

Приведенное выше построение канонических $x$-зависимых переменных является наиболее простым. В некоторых задачах может оказаться полезным другой набор канонических переменных. Построим этот набор координат, обозначаемых $\zeta_{j}\left(x_{i}\right)$ :

$$
\zeta_{j}\left(x_{i}\right)=\int d^{3} \xi \xi_{j} \delta\left(\vec{x}-\vec{x}\left(\xi_{i}, t\right)\right) .
$$

Вычисляя скобки Пуассона величин $\zeta_{j}\left(x_{i}\right)$ и $l_{j}\left(x_{i}\right)$, получаем

$$
\left\{l_{j}\left(x_{i}\right), \zeta_{k}\left(y_{i}\right)\right\}=\zeta_{k}\left(x_{i}\right) \frac{\partial}{\partial x_{j}} \delta(\vec{x}-\vec{y}) .
$$

Отсюда заключаем, что величины $l_{j}\left(x_{i}\right)$ должны иметь следуюшее представление:

$$
l_{j}\left(x_{i}\right)=\zeta_{k}\left(x_{i}\right) \frac{\partial}{\partial x_{j}} \eta_{k}\left(x_{i}\right),
$$

где $\eta_{k}\left(x_{i}\right)$ - переменная, канонически сопряженная с $\zeta_{j}\left(x_{i}\right)$ :

$$
\left\{\eta_{j}\left(x_{i}\right), \zeta_{k}\left(y_{i}\right)\right\}=\delta_{j k} \delta(\vec{x}-\vec{y}) .
$$


Канонический гамильтониан (22) должен быть выражен теперь через новые переменные $\left(\pi_{m}\left(x_{i}\right), \xi_{k}\left(y_{i}\right)\right)$. Действительно, введем единицу

$$
1=\int d^{3} x \delta\left(\vec{x}-\vec{x}\left(\xi_{i}\right)\right)
$$

в подынтегральное выражение в (22) и изменим порядок интегрирования:

$$
H=\int d^{3} x \int d^{3} \xi\left(\frac{1}{2 m} \vec{p}^{2}\left(\xi_{i}, t\right)+f\left(\operatorname{det} A\left(\xi_{i}\right)\right)\right) \delta\left(\vec{x}-\vec{x}\left(\xi_{i}\right)\right) .
$$

Выполняя интегрирование по $\xi$ с помощью (2) и (24), получаем

$$
H=\int d^{3} x\left(\frac{1}{2 m \rho\left(x_{i}\right)} \vec{l}^{2}\left(x_{i}\right)+\rho\left(x_{i}\right) f\left(\frac{1}{\rho\left(x_{i}\right)}\right)\right) .
$$

Воспользовавшись равенством (37), можно выразить гамильтониан $H$ через канонические переменные $\left(\pi_{m}\left(x_{i}\right), \xi_{k}\left(y_{i}\right)\right)$ :

$$
H=\int d^{3} x\left(\frac{1}{2 m \rho\left(x_{i}\right)} \frac{\partial \xi_{k}\left(x_{i}\right)}{\partial x_{j}} \frac{\partial \xi_{m}\left(x_{i}\right)}{\partial x_{j}} \pi_{k}\left(x_{i}\right) \pi_{m}\left(x_{i}\right)+V\left(\rho\left(x_{i}\right)\right)\right)
$$

где введено обозначение $V\left(\rho\left(x_{i}\right)\right)$ для “потенциальной” части энергии. Этот член представляет внутреннюю энергию жидкости и должен обрашаться в нуль (с точностью до несушественной константы) для однородного распределения плотности $\rho\left(x_{i}\right)=\rho_{0}$. Феноменологическое выражение для $V\left(\rho\left(x_{i}\right)\right)$ может быть записано следуюшим образом [6]:

$$
V\left(\rho\left(x_{i}\right)\right)=\frac{\kappa}{2 \rho_{0}}\left(\delta \rho\left(x_{i}\right)\right)^{2}+\lambda\left(\nabla \rho\left(x_{i}\right)\right)^{2}+\cdots,
$$

где $\delta \rho\left(x_{i}\right)$ - отклонение плотности от однородного распределения:

$$
\delta \rho\left(x_{i}\right)=\rho\left(x_{i}\right)-\rho_{0} .
$$

Первый член в выражении (47) отвечает за звуковые волны в жидкости ( $\kappa$ - скорость звука), а второй член описывает дисперсию звуковых волн.

Для того чтобы обнаружить связь порождаемого (46) гамильтонова потока с геодезическим потоком [7], введем метрический тензор

$$
g_{j k}\left(x_{i}\right)=A_{j}^{m}\left(\xi_{i}\left(x_{k}\right)\right) A_{k}^{m}\left(\xi_{i}\left(x_{k}\right)\right) .
$$

В этих обозначениях гамильтониан (46) принимает вид

$$
H=\int d^{3} x\left(\frac{1}{2 m} \sqrt{g\left(x_{i}\right)} g^{k m}\left(x_{i}\right) \pi_{k}\left(x_{i}\right) \pi_{m}\left(x_{i}\right)+V\left(\frac{1}{\sqrt{g\left(x_{i}\right)}}\right)\right) .
$$


Метрический тензор с верхними индексами $g^{j k}\left(x_{i}\right)$ обозначает, как обычно, обратную матрицу, и

$$
g\left(x_{i}\right)=\operatorname{det} g_{j k}\left(x_{i}\right)=\frac{1}{\rho^{2}\left(x_{i}\right)} .
$$

Представление (50) дает возможность рассматривать гидродинамику как геодезический поток на динамическом многообразии с метрикой $g_{j k}\left(x_{i}\right)$, и это обстоятельство дает возможность вывести многие обшие свойства динамики жидкости (см., например, [7]).

Завершим данный раздел одним важным замечанием относительно нашего описания гидродинамики. В рамках настояшего рассмотрения фазовое пространство трехмерной жидкости является шестимерным, что естественно вытекает из принятого нами подхода. Это можно сравнить с недавней работой [8], где (в наших обозначениях) только $\rho\left(x_{i}\right)$ и $v_{j}\left(x_{i}\right)$ рассматриваются как координаты в фазовом пространстве. Такая точка зрения представлена также в различных учебниках и статьях (см., например, [7], [6], [9]).

Согласно обычной точке зрения состояние жидкости определяется ее скоростью и плотностью, поэтому все остальные переменные, подобные нашим $\vec{x}\left(\xi_{i}\right)$, не нужны. Действительно, решая уравнения движения Эйлера, можно выразить скорость $\vec{v}\left(x_{i}, t\right)$ и плотность $\rho\left(x_{i}, t\right)$ в момент времени $t$ через начальные данные $\left.\vec{v}\left(x_{i}, t\right)\right|_{t=0}$ и $\left.\rho\left(x_{i}, t\right)\right|_{t=0}$. Далее, из определения (3) величины $\vec{v}\left(x_{i}, t\right)$ следует

$$
\vec{v}(x(\xi, t), t)=\dot{\vec{x}}(\xi, t)
$$

(здесь мы для краткости опустили индексы при аргументах). Очевидно, можно решить эти уравнения относительно $\vec{x}(\xi, t)$, если известны начальные данные $\left.\vec{x}(\xi, t)\right|_{t=0}$. Поэтому может показаться, что переменные $\vec{x}(\xi, t)$ являются лишними, так как они могут быть получены из других переменных. Однако начальные данные $\left.\vec{x}(\xi, t)\right|_{t=0}$ суть половина канонических переменных гамильтонова формализма. Таким образом, при используемом подходе нам необходимо больше переменных. Эти дополнительные переменные обеспечивают полное описание жидкости в том смысле, что, решая уравнения движения, мы определяем не только величины $\vec{v}\left(x_{i}, t\right)$ и $\rho\left(x_{i}, t\right)$, но и траектории частиц, которые не могут быть получены в рамках традиционного формализма. Эта ситуация аналогична врашению твердого тела: здесь фазовое пространство образовано тремя углами и тремя компонентами момента импульса $J_{i}$. Поскольку гамильтониан зависит только от компонент $J_{i}$, эволюция момента импульса может рассматриваться отдельно. Такое описание для этой механической системы является неполным. Полное описание непременно должно включать эволюцию углов, которые определяют положение твердого тела в пространстве.

В случае динамики жидкости полное описание должно производиться в шестимерном фазовом пространстве $\Gamma$, которое образовано переменными $\vec{x}(\xi)$ и $\vec{p}(\xi)$ (или $\vec{\xi}(x)$ и $\vec{l}(x))$. Если, как это обычно бывает, гамильтониан зависит только от величин $\vec{l}(x)$ и $\rho(x)$, возможно неполное описание в терминах скоростей и плотностей, рассматриваемых как "существенные" переменные. Это означает, что мы не интересуемся эволюцией тех координат в фазовом пространстве, которые рассматриваются как несущественные. "Сушественные" координаты не обязательно образуют симплектическое подпространство 
в Г. Невырожденные в Г скобки Пуассона могут стать вырожденными на подпространстве в $Г$, соответствующем “существенным" переменным. Такое положение в самом деле имеет место в случаях твердого тела и в традиционной гидродинамике. В первом случае вырожденность алгебры скобок Пуассона для "существенных" переменных (момента импульса) хорошо известна. Ее центральный элемент - оператор Казимира группы врашений. В случае гидродинамики алгебра “существенных" переменных (скоростей и плотностей) имеет следующий вид:

$$
\begin{aligned}
\left\{v_{j}\left(x_{i}\right), v_{k}\left(y_{i}\right)\right\} & =-\frac{1}{\rho(x)}\left(\nabla_{j} v_{k}\left(x_{i}\right)-\nabla_{k} v_{j}\left(x_{i}\right)\right) \delta(\vec{x}-\vec{y}), \\
\left\{v_{j}\left(x_{i}\right), \rho\left(y_{i}\right)\right\} & =\nabla_{j} \delta(\vec{x}-\vec{y}) \\
\left\{\rho\left(x_{i}\right), \rho\left(y_{i}\right)\right\} & =0 .
\end{aligned}
$$

Данная алгебра может быть получена из равенств (25), (30) и (31). Центр этой алгебры является бесконечномерным, и его структура зависит от размерности $x$-пространства. В двумерном случае центр образован элементами

$$
I_{k}=\int d x d y\left(\frac{\partial v_{1}(x, y)}{\partial y}-\frac{\partial v_{2}(x, y)}{\partial x}\right)^{k} \rho^{1-k}(x, y)
$$

(подробное обсуждение см. в [7], [8]). В трехмерном случае общий ответ авторам не известен, и его получение выходит за рамки настоящей работы. В качестве примера мы упомянем, что инвариант Хопфа

$$
Q=\int d^{3} x \epsilon_{j k l} v_{j}(\vec{x}) \nabla_{k} v_{l}(\vec{x})
$$

принадлежит этому центру.

\section{4. БЕСКОНЕЧНОМЕРНАЯ СИММЕТРИЯ И ИНТЕГРАЛЫ ДВИЖЕНИЯ}

Как упоминалось выше, лагранжиан (17) обладает инвариантностью относительно “сохраняюшей объем” группы диффеоморфизмов $\operatorname{SDiff}[D]$, где $D \subseteq \mathbb{R}^{3}$. Действительно, запишем лагранжиан (17) через плотность лагранжиана:

$$
L=\int d^{3} \xi \mathcal{L}\left(\xi_{i}\right)
$$

и рассмотрим преобразования координат $\xi_{i} \in D$ из $\operatorname{SDiff}[D]$

$$
\begin{gathered}
\xi_{j} \rightarrow \xi_{j}^{\prime}=\phi_{j}\left(\xi_{i}\right), \\
\operatorname{det} \frac{\partial \phi_{j}\left(\xi_{i}\right)}{\partial \xi_{k}}=1 .
\end{gathered}
$$


Вследствие (58), очевидно, имеем

$$
L=\int d^{3} \phi\left(\xi_{i}\right) \mathcal{L}\left(\phi\left(\xi_{i}\right)\right)=\int d^{3} \xi \mathcal{L}\left(\phi\left(\xi_{i}\right)\right),
$$

и согласно теореме Нётер эта инвариантность приводит к сушествованию бесконечного множества интегралов движения. Чтобы получить эти интегралы, необходимо прежде всего найти параметризацию преобразований $(57),(58)$ в окрестности тождественного преобразования

$$
\phi_{j}\left(\xi_{i}\right)=\xi_{j}+\alpha_{j}\left(\xi_{i}\right) .
$$

Из условия (58) вытекает уравнение для $\alpha_{j}\left(\xi_{i}\right)$ :

$$
\frac{\partial \alpha_{j}\left(\xi_{i}\right)}{\partial \xi_{j}}=0
$$

Далее необходимо явно учесть, что сохраняюший объем диффеоморфизм (57) оставляет границу области $D$ инвариантной. Мы ограничимся тем случаем, когда область $D$ получена в результате исключения из пространства $\mathbb{R}^{3}$ области с дифференцируемой границей, заданной условием

$$
g\left(\xi_{i}\right)=0 .
$$

Физически это означает, что мы помешаем в жидкость фиксированное тело, форма которого задается формулой (62). В этом случае условие того, что инфинитезимальный диффеоморфизм (60) сохраняет область $D$, есть

$$
\left.g\left(\xi_{j}+\alpha_{j}\left(\xi_{i}\right)\right)\right|_{g\left(\xi_{i}\right)=0}=0,
$$

или

$$
\left.\alpha_{j}\left(\xi_{i}\right) \nabla_{j} g\left(\xi_{i}\right)\right|_{g\left(\xi_{i}\right)=0}=0 .
$$

Геометрически уравнение (64) означает, что вектор $\vec{\alpha}\left(\xi_{i}\right)$ является касательным к определяемой формулой (62) поверхности, поскольку вектор $\left.\nabla_{j} g\left(\xi_{i}\right)\right|_{g\left(\xi_{i}\right)=0}$ пропорционален нормали $n_{j}\left(\xi_{i}\right)$ к поверхности $(62)$ в точке $\xi_{i}$.

Из теоремы Нётер получаем, что из инвариантности лагранжиана относительно преобразования (60) вытекает следуюший закон сохранения:

$$
\frac{\partial}{\partial t} \int_{D} d^{3} \xi p_{m}\left(\xi_{i}\right) \frac{\partial x_{m}\left(\xi_{i}\right)}{\partial \xi_{l}} \alpha_{l}\left(\xi_{i}\right)=0
$$

где $\alpha_{l}\left(\xi_{i}\right)$ удовлетворяет условиям (61) и (64). Наличие этих условий препятствует взятию вариации от левой части $(65)$ по $\alpha_{l}\left(\xi_{i}\right)$ и получению интегралов движения в локальной форме.Для этого необходимо получить из условий (61) и (64) интегральные свойства $\alpha_{l}\left(\xi_{i}\right)$. 
Рассмотрим произвольную однозначную дифференцируемую в области $D$ функцию $\beta\left(\xi_{i}\right)$. Тогда имеют место следуюшие равенства:

$$
0=\int_{D} d^{3} \xi \beta\left(\xi_{i}\right) \frac{\partial \alpha_{j}\left(\xi_{i}\right)}{\partial \xi_{j}}=\int_{D} d^{3} \xi \frac{\partial}{\partial \xi_{j}}\left(\beta\left(\xi_{i}\right) \alpha_{j}\left(\xi_{i}\right)\right)-\int_{D} d^{3} \xi \alpha_{j}\left(\xi_{i}\right) \frac{\partial \beta\left(\xi_{i}\right)}{\partial \xi_{j}}
$$

Первое равенство справедливо вследствие условия (61). Используя теорему Стокса, можно преобразовать интеграл от полной производной в последнем равенстве (66):

$$
\int_{D} d^{3} \xi \frac{\partial}{\partial \xi_{j}}\left(\beta\left(\xi_{i}\right) \alpha_{j}\left(\xi_{i}\right)\right)=\int_{\partial D} d S_{j}\left(\beta\left(\xi_{i}\right) \alpha_{j}\left(\xi_{i}\right)\right)=0,
$$

где $\partial D$ обозначает границу $D$. Последний интеграл в (67) обрашается в нуль в силу условия (64), поскольку дифференциал $d S_{j}$ пропорционален вектору нормали к поверхности $\partial D$, определенной формулой (62). Из равенств (66) и (67) мы заключаем, что

$$
\int_{D} d^{3} \xi \alpha_{j}\left(\xi_{i}\right) \frac{\partial \beta\left(\xi_{i}\right)}{\partial \xi_{j}}=0
$$

для любой гладкой дифференцируемой функции $\beta\left(\xi_{i}\right)$. Учитывая данное свойство $\alpha_{j}\left(\xi_{i}\right)$, из законов сохранения (65) получаем, что величины

$$
J_{k}\left(\xi_{i}\right)=p_{m}\left(\xi_{i}\right) \frac{\partial x_{m}\left(\xi_{i}\right)}{\partial \xi_{k}}
$$

сохраняются по модулю некоторого члена, который является градиентом от скаляра. В частности, это означает, что величины

$$
R_{j}\left(\xi_{i}\right)=\epsilon_{j k l} \frac{\partial}{\partial \xi_{k}} J_{l}\left(\xi_{i}\right)=\epsilon_{j k l} \frac{\partial}{\partial \xi_{k}}\left(p_{m}\left(\xi_{i}\right) \frac{\partial x_{m}\left(\xi_{i}\right)}{\partial \xi_{l}}\right)
$$

являются интегралами движения. Заметим, что, поскольку группа инвариантности является бесконечномерной, мы получили бесконечное число интегралов движения. По отношению к скобкам Пуассона $(23)$ величины $R_{j}\left(\xi_{i}\right)$ образуют алгебру. Эта алгебра может быть выписана в компактном виде для интегральных объектов

$$
R[\phi]=\int d^{3} \xi \phi_{j}\left(\xi_{i}\right) R_{j}\left(\xi_{i}\right),
$$

где $\phi_{j}\left(\xi_{i}\right)$ - гладкие быстроубывающие функции. Индуцированная скобками Пуассона (23) алгебра $R[\phi]$ имеет вид

$$
\{R[\phi], R[\psi]\}=R[\operatorname{curl} \phi \times \operatorname{curl} \psi] .
$$

Построение $x$-зависимого объекта, соответствуюшего $R_{j}\left(\xi_{i}\right)$, представляет собой непростую задачу, поскольку наше "усреднение" с $\delta\left(\vec{x}-\vec{x}\left(\xi_{i}\right)\right)$ приведет к появлению временно́й зависимости, и вместо сохраняюшегося объекта мы получим некоторую плотность, производная по времени которой дает дивергенцию “тока”. Поэтому необходимо ввести усреднение другого рода без явного обрашения к координатам $\vec{x}\left(\xi_{i}\right)$. С этой 
целью напомним, что под действием диффеоморфизма замкнутый контур переходит в замкнутый контур. Рассмотрим такой контур $\lambda$ и поверхность $\sigma$, границей которой он является. Интеграл

$$
V=\int_{\sigma} d S_{j} R_{j}\left(\xi_{i}\right)
$$

в котором вектор $d S_{j}$, как обычно, представляет собой элемент плошади, умноженный на вектор единичной нормали к поверхности, сохраняется, поскольку сохраняется вектор $R_{j}\left(\xi_{i}\right)$. Далее, из теоремы Стокса имеем

$$
V=\oint_{\lambda} d \xi_{j} p_{m}\left(\xi_{i}\right) \frac{\partial x_{m}\left(\xi_{i}\right)}{\partial \xi_{j}}
$$

Делая замену переменных в (74), получаем

$$
V=\oint_{\Lambda} d x_{j} \frac{l_{j}\left(x_{i}\right)}{\rho\left(x_{i}\right)}=\oint_{\Lambda} d x_{j} v_{j}\left(x_{i}\right)
$$

где $\Lambda$ - образ контура $\lambda$ под действием диффеоморфизма $\xi_{j} \rightarrow x_{j}\left(\xi_{i}\right)$. Величина (75) очень хорошо известна в гидродинамике как "циркуляция", и ее сохранение известно как теорема Томсона [10]. Связь сохранения циркуляции с инвариантностью относительно специальных диффеоморфизмов впервые была установлена в явном виде в работе [11], хотя этот результат мог также быть извлечен из обшего рассмотрения в приложении 2 книги [7].

Сохранение циркуляции - не единственное следствие (65). Рассмотрим, например, случай двумерного пространства. Здесь вместо сохраняющегося вектора $R_{j}\left(\xi_{i}\right)$ мы будем иметь сохраняюшийся скаляр

$$
R\left(\xi_{i}\right)=\epsilon_{k l} \frac{\partial}{\partial \xi_{k}} J_{l}\left(\xi_{i}\right)=\epsilon_{k l} \frac{\partial}{\partial \xi_{k}}\left(p_{m}\left(\xi_{i}\right) \frac{\partial x_{m}\left(\xi_{i}\right)}{\partial \xi_{l}}\right) .
$$

Этот скаляр определяет следуюшие интегралы движения:

$$
I_{n}=\int_{D} d^{2} \xi R^{n}\left(\xi_{i}\right)=\int_{D} d^{2} \xi\left(\epsilon_{k l} \frac{\partial}{\partial \xi_{k}}\left(p_{m}\left(\xi_{i}\right) \frac{\partial x_{m}\left(\xi_{i}\right)}{\partial \xi_{l}}\right)\right)^{n}
$$

Делая в (77) замену переменных $\xi_{j} \rightarrow \xi_{j}\left(x_{i}\right)$, что возможно, поскольку $\xi_{j}\left(x_{i}\right)$ есть дифффеоморфизм области $D$, получаем

$$
I_{n}=\int_{D} d^{2} x \rho\left(x_{i}\right)\left(\epsilon_{k l} A_{k}^{j}(\xi(x)) A_{l}^{m}(\xi(x)) \frac{\partial p_{m}(\xi(x))}{\partial x_{j}}\right)^{n},
$$

где матрица $A_{k}^{j}(\xi)$ определена формулой (14). Интегралы $I_{n}$ можно представить как

$$
I_{n}=\int_{D} d^{2} x \rho\left(x_{i}\right)^{1-n}\left(\epsilon_{j m} \frac{\partial}{\partial x_{j}} \frac{l_{m}\left(x_{i}\right)}{\rho\left(x_{i}\right)}\right)^{n}
$$


если использовать следующее свойство двумерной матрицы $A_{k}^{j}(\xi)$ :

$$
\epsilon_{k l} A_{k}^{j}(\xi(x)) A_{l}^{m}(\xi(x))=\epsilon_{j m} \operatorname{det} A(\xi(x))=\frac{\epsilon_{j m}}{\rho\left(x_{i}\right)} .
$$

Напомним, что в случае лагранжиана (17) мы рассматриваем

$$
\frac{l_{j}\left(x_{i}\right)}{\rho\left(x_{i}\right)}=m v_{j}\left(x_{i}\right) \text {. }
$$

Поэтому интегралы $I_{n}$ совпадают с центром (54) алгебры Пуассона (53).

В трехмерном пространстве можно построить аналогичные интегралы движения, интегрируя произведения векторов $(70)$ :

$$
K_{j_{1}, j_{2}, \ldots, j_{n}}=\int_{D} d^{3} \xi R_{j_{1}}\left(\xi_{i}\right) R_{j_{2}}\left(\xi_{i}\right) \ldots R_{j_{n}}\left(\xi_{i}\right)
$$

Делая замену переменных $\xi_{j} \rightarrow \xi_{j}\left(x_{i}\right)$, как и вьше, получим

$$
R_{j}(\xi) \rightarrow R_{j}(\xi(x))=\epsilon_{j k l} A_{k}^{m}(\xi(x)) A_{l}^{n}(\xi(x)) \frac{\partial p_{n}(\xi(x))}{\partial x_{m}}
$$

В трехмерном случае матрицы $A_{j}^{m}(\xi(x))$ удовлетворяют уравнению

$$
\epsilon_{j k l} A_{k}^{m}(\xi(x)) A_{l}^{n}(\xi(x))=\epsilon_{m n r} \frac{\partial \xi_{j}(x)}{\partial x_{r}} \operatorname{det} A(\xi(x)) .
$$

Поэтому (83) принимает вид

$$
R_{j}(\xi(x))=\frac{1}{\rho\left(x_{i}\right)} \epsilon_{m n r} \frac{\partial \xi_{j}(x)}{\partial x_{r}} \frac{\partial p_{n}(\xi(x))}{\partial x_{m}} .
$$

Интегралы $K_{n}$ в выражении (82) преврашаются в

$$
K_{j_{1}, j_{2}, \ldots, j_{n}}=\int_{D} d^{3} x \rho\left(x_{i}\right) R_{j_{1}}(\xi(x)) R_{j_{2}}(\xi(x)) \ldots R_{j_{n}}(\xi(x)) .
$$

Очевидно, из-за наличия выражения $\partial \xi_{j}(x) / \partial x_{r}$ в $(85)$ в этом случае невозможно выразить (85) только в терминах скорости и плотности, т.е. эйлерово описание не допускает интегралов движения такого рода.

В трехмерном случае сушествует еще один интеграл, который не существует в любой другой размерности. Напомним, что вектор $J_{k}\left(\xi_{i}\right)$, заданньй формулой $(69)$, сохраняется с точностью до градиента, следовательно, интеграл

$$
Q=\int_{D} d^{3} \xi J_{k}\left(\xi_{i}\right) R_{k}\left(\xi_{i}\right)
$$

сохраняется, поскольку $\vec{R}\left(\xi_{i}\right)=\operatorname{curl} \vec{J}\left(\xi_{i}\right)$. Преобразуя $\xi_{j}$-зависимые переменные в $x_{j}$-зависимые, получаем инвариант Хопфа

$$
Q=\int_{D} d^{3} x \epsilon_{j k l} p_{j}(\xi(x)) \frac{\partial p_{k}(\xi(x))}{\partial x_{l}} .
$$




\section{5. ВКЛЮЧЕНИЕ ЭЛЕКТРОМАГНИТНОГО ВЗАИМОДЕЙСТВИЯ. ПЛАЗМА}

Будем рассматривать плазму как жидкость, состоящую из двух компонентов, а именно электронов с массой $m$ и электрическим зарядом $-e$ и ионов с массой $M$ и зарядом $+e$. Координаты электронов будем обозначать как $\vec{x}\left(\xi_{i}\right)$, а координаты ионов - как $\vec{X}\left(\Xi_{i}\right)$. Взаимодействие компонентов плазмы с электромагнитным полем $A_{\mu}(x)$ определяется лагранжианом

$$
\begin{aligned}
L= & L_{0}^{\mathrm{el}}+L_{0}^{\mathrm{ion}}+e \int d^{3} \xi\left[A_{0}\left(\vec{x}\left(\xi_{i}, t\right)\right)-\dot{\vec{x}}\left(\xi_{i}, t\right) \vec{A}\left(\vec{x}\left(\xi_{i}, t\right)\right)\right]- \\
& -e \int d^{3} \Xi\left[A_{0}\left(\vec{x}\left(\Xi_{i}, t\right)\right)-\dot{\vec{X}}\left(\Xi_{i}, t\right) \vec{A}\left(\vec{X}\left(\Xi_{i}, t\right)\right)\right]-\frac{1}{4} \int d^{3} x F^{\mu \nu}(x, t) F_{\mu \nu}(x, t),
\end{aligned}
$$

где $L_{0}^{\mathrm{el}}$ и $L_{0}^{\mathrm{ion}}$ - "свободные" лагранжианы:

$$
\begin{aligned}
L_{0}^{\mathrm{el}} & =\int d^{3} \xi\left[\frac{m \dot{\vec{x}}^{2}\left(\xi_{i}, t\right)}{2}-f^{\mathrm{el}}\left(\operatorname{det} \frac{\partial x_{j}\left(\xi_{i}, t\right)}{\partial \xi_{k}}\right)\right], \\
L_{0}^{\mathrm{ion}} & =\int d^{3} \Xi\left[\frac{M \dot{\vec{X}}^{2}\left(\Xi_{i}, t\right)}{2}-f^{\mathrm{ion}}\left(\operatorname{det} \frac{\partial X_{j}\left(\Xi_{i}, t\right)}{\partial \Xi_{k}}\right)\right],
\end{aligned}
$$

и $F_{\mu \nu}(x, t)$ обозначает тензор электромагнитного поля

$$
F_{\mu \nu}(x, t)=\partial_{\mu} A_{\nu}(x, t)-\partial_{\nu} A_{\mu}(x, t) .
$$

Преимушество лагранжева описания жидкости (плазмы) очевидно. Использование координат заряженных частиц в качестве основных переменных делает явным взаимодействие с электромагнитным полем.

Лагранжиан (89) обладает $U(1)$-калибровочной инвариантностью. Мы будем использовать обычный гамильтонов формализм для систем со связями [12]. Канонические переменные суть

$$
\begin{aligned}
\vec{x}\left(\xi_{i}\right), & \vec{p}\left(\xi_{i}\right)=\frac{\delta L}{\delta \dot{\vec{x}}\left(\xi_{i}\right)} ; \\
\vec{X}\left(\Xi_{i}\right), & \vec{P}\left(\Xi_{i}\right)=\frac{\delta L}{\delta \dot{\vec{X}}\left(\Xi_{i}\right)} ; \\
\vec{A}\left(x_{i}\right), & \vec{P}_{\mathrm{em}}\left(x_{i}\right)=\frac{\delta L}{\delta \dot{\vec{A}}\left(x_{i}\right)}=-\vec{E}\left(x_{i}\right) ; \\
A_{0}\left(x_{i}\right), & P_{\mathrm{em}}^{0}\left(x_{i}\right)=0,
\end{aligned}
$$

где $\vec{E}\left(x_{i}\right)$ - напряженность электрического поля,

$$
\vec{E}\left(x_{i}\right)=-\nabla A_{0}\left(x_{i}\right)-\dot{\vec{A}}\left(x_{i}\right)
$$


Последнее из выражений (92) является в действительности первичной связью. Преобразование Лежандра лагранжиана (89) дает канонический гамильтониан

$$
\begin{aligned}
H= & \int d^{3} x\left[\frac{1}{2}\left(\vec{P}_{\mathrm{em}}^{2}\left(x_{i}\right)+\vec{H}^{2}\left(x_{i}\right)\right)+A_{0}\left(x_{i}\right) \nabla \vec{P}_{\mathrm{em}}\left(x_{i}\right)\right]+ \\
& +\int d^{3} \xi\left[\frac{1}{2 m}\left(\vec{p}\left(\xi_{i}\right)+e \vec{A}\left(x\left(\xi_{i}\right)\right)\right)^{2}-e A_{0}\left(x\left(\xi_{i}\right)\right)+f^{\mathrm{el}}\left(\operatorname{det} \frac{\partial x_{j}\left(\xi_{i}, t\right)}{\partial \xi_{k}}\right)\right]+ \\
+ & \int d^{3} \Xi\left[\frac{1}{2 M}\left(\vec{P}\left(\Xi_{i}\right)-e \vec{A}\left(X\left(\Xi_{i}\right)\right)\right)^{2}+e A_{0}\left(X\left(\Xi_{i}\right)\right)+f^{\operatorname{ion}}\left(\operatorname{det} \frac{\partial X_{j}\left(\Xi_{i}, t\right)}{\partial \Xi_{k}}\right)\right],
\end{aligned}
$$

где $\vec{H}\left(x_{i}\right)=\operatorname{curl} \vec{A}\left(x_{i}\right)$ - напряженность магнитного поля. Условие сохранения первичной связи $P_{\mathrm{em}}\left(x_{i}\right)=0$ дает вторичную связь (закон Гаусса)

$$
\nabla_{j}\left(P_{\mathrm{em}}\left(x_{i}\right)\right)_{j}-e\left(\rho_{\mathrm{el}}\left(x_{i}\right)-\rho_{\mathrm{ion}}\left(x_{i}\right)\right)=0 .
$$

Теперь к первичной связи $P_{\mathrm{em}}^{0}\left(x_{i}\right)=0$ можно добавить калибровочное условие $A_{0}\left(x_{i}\right)=0$ и исключить эти переменные из рассмотрения. Вводя $x$-зависимые функции вместо $\xi$-зависимых и $\Xi$-зависимых, как это было сделано в разделе 2 , мы получаем гамильтониан плазмы в следующем виде:

$$
\begin{aligned}
H= & \int d^{3} x\left[\frac{1}{2}\left(\vec{P}_{\mathrm{em}}^{2}\left(x_{i}\right)+\vec{H}^{2}\left(x_{i}\right)\right)+\frac{\left(\vec{l}\left(x_{i}\right)+e \rho_{\mathrm{el}}\left(x_{i}\right) \vec{A}\left(x_{i}\right)\right)^{2}}{2 m \rho_{\mathrm{el}}\left(x_{i}\right)}+\right. \\
& \left.+\frac{\left(\vec{L}\left(x_{i}\right)+e \rho_{\mathrm{ion}}\left(x_{i}\right) \vec{A}\left(x_{i}\right)\right)^{2}}{2 m \rho_{\mathrm{ion}}\left(x_{i}\right)}+v_{\mathrm{el}}\left(\rho_{\mathrm{el}}\left(x_{i}\right)\right)+v_{\mathrm{ion}}\left(\rho_{\mathrm{ion}}\left(x_{i}\right)\right)\right],
\end{aligned}
$$

где введены обозначения

$$
\begin{aligned}
\vec{l}\left(x_{i}\right) & =\int d^{3} \xi \vec{p}\left(\xi_{i}\right) \delta\left(\vec{x}-\vec{x}\left(\xi_{i}\right)\right), \\
\vec{L}\left(x_{i}\right) & =\int d^{3} \Xi \vec{P}\left(\Xi_{i}\right) \delta\left(\vec{x}-\vec{X}\left(\Xi_{i}\right)\right) .
\end{aligned}
$$

Этот гамильтониан калибровочно-инвариантен относительно преобразований, порождаемых связью (95). Далее мы наложим кулоновское калибровочное условие на электромагнитное поле:

$$
\nabla_{j} A_{j}\left(x_{i}\right)=0,
$$

и, следуя обычной процедуре, исключим продольные составляюшие векторов $\vec{A}\left(x_{i}\right)$ и $\vec{P}_{\mathrm{em}}\left(x_{i}\right)$. В результате фиксации калибровки продольная часть $\vec{P}_{\mathrm{em}}\left(x_{i}\right)$ приводит к появлению кулоновского члена в гамильтониане

$$
H_{\mathrm{Col}}=-e^{2} \int d^{3} x\left(\rho_{\mathrm{el}}\left(x_{i}\right)-\rho_{\mathrm{ion}}\left(x_{i}\right)\right)\left(-\frac{1}{4 \pi|\vec{x}-\vec{y}|}\right)\left(\rho_{\mathrm{el}}\left(y_{i}\right)-\rho_{\mathrm{ion}}\left(y_{i}\right)\right),
$$


и в целом гамильтониан принимает следующий вид:

$$
\begin{aligned}
H= & H_{\mathrm{Col}}+\int d^{3} x\left[\frac{1}{2}\left(\vec{P}_{\mathrm{em} \perp}^{2}\left(x_{i}\right)+\vec{H}^{2}\left(x_{i}\right)\right)+\right. \\
& +\frac{\left(\vec{l}\left(x_{i}\right)+e \rho_{\mathrm{el}}\left(x_{i}\right) \vec{A}_{\perp}\left(x_{i}\right)\right)^{2}}{2 m \rho_{\mathrm{el}}\left(x_{i}\right)}+v_{\mathrm{el}}\left(\rho_{\mathrm{el}}\left(x_{i}\right)\right)+ \\
& \left.+\frac{\left(\vec{L}\left(x_{i}\right)+e \rho_{\mathrm{ion}}\left(x_{i}\right) \vec{A}_{\perp}\left(x_{i}\right)\right)^{2}}{2 m \rho_{\mathrm{ion}}\left(x_{i}\right)}+v_{\mathrm{ion}}\left(\rho_{\mathrm{ion}}\left(x_{i}\right)\right)\right],
\end{aligned}
$$

где нижний индекс $\perp$ используется для обозначения поперечных составляюших электромагнитных переменных, для которых скобки Пуассона (Дирака) даются выражениeм $[12]$

$$
\left\{P_{\perp j}^{\mathrm{em}}(x), A_{\perp k}(y)\right\}=\left(\delta_{j k}-\frac{1}{\Delta} \partial_{j} \partial_{k}\right) \delta(\vec{x}-\vec{y})
$$

Как обычно, кулоновский член (99) содержит бесконечную аддитивную часть, связанную с “самодействием”, которая должна быть вычтена. Известно, что в равновесной плазме кулоновское взаимодействие экранируется облаком и остаточное взаимодействие Дебая является короткодействующим (см., например, [13]). В [14] было установлено, что кулоновский член сводится к локальному функционалу разности плотностей зарядов $\rho_{\mathrm{el}}\left(x_{i}\right)-\rho_{\text {ion }}\left(x_{i}\right)$.

Следуя нашему рассмотрению жидкости в разделе 3 , можно ввести канонические координаты $\vec{\xi}\left(x_{i}\right), \vec{\pi}\left(x_{i}\right)$ для электронного компонента и $\vec{\Xi}\left(x_{i}\right), \vec{\Pi}\left(x_{i}\right)$ для ионного компонента плазмы:

$$
\begin{aligned}
\left\{\pi_{j}\left(x_{i}\right), \xi_{k}\left(y_{i}\right)\right\} & =\delta_{j k} \delta(\vec{x}-\vec{y}), \\
\left\{\Pi_{j}\left(x_{i}\right), \Xi_{k}\left(y_{i}\right)\right\} & =\delta_{j k} \delta(\vec{x}-\vec{y}),
\end{aligned}
$$

для которых

$$
l_{j}\left(x_{i}\right)=\vec{\xi}\left(x_{i}\right) \frac{\partial \vec{\pi}\left(x_{i}\right)}{\partial x_{j}}, \quad L_{j}\left(x_{i}\right)=\vec{\Xi}\left(x_{i}\right) \frac{\partial \vec{\Pi}\left(x_{i}\right)}{\partial x_{j}} .
$$

Подставляя (103) в (100), получаем гамильтониан в терминах канонических переменных.

Лагранжиан (89) инвариантен относительно диффеоморфизмов, сохраняющих объем обоих компонентов плазмы в отдельности, так что в данном случае мы имеем два набора законов сохранения - один для электронов, другой для ионов. Применяя описанную в предыдущем разделе процедуру, получаем сохраняющиеся циркуляции

$$
V_{\Lambda}^{\mathrm{el}}=\oint_{\Lambda} d x_{j} \frac{l_{j}\left(x_{i}\right)}{\rho_{\mathrm{el}}\left(x_{i}\right)}, \quad V_{\Lambda}^{\mathrm{ion}}=\oint_{\Lambda} d x_{j} \frac{L_{j}\left(x_{i}\right)}{\rho_{\mathrm{ion}}\left(x_{i}\right)}
$$

где $l_{j}(x)$ и $L_{j}(x)$ даются выражениями (103). Подобным способом можно построить аналоги интегралов (86) и (88) для рассматриваемого случая. Заметим, что в случае 
плазмы равенство (38) не справедливо из-за наличия электромагнитного поля, вместо него имеют место равенства

$$
\begin{aligned}
\vec{l}(x) & =\rho_{\mathrm{el}}(x)\left(m \vec{v}_{\mathrm{el}}(x)-e \vec{A}(x)\right), \\
\vec{L}(x) & =\rho_{\mathrm{ion}}(x)\left(M \vec{v}_{\mathrm{ion}}(x)+e \vec{A}(x)\right),
\end{aligned}
$$

поэтому выражения (38) не являются циркуляциями скоростей. В то же время, складывая циркуляции $V_{\Lambda}^{\mathrm{el}}$ и $V_{\Lambda}^{\text {ion }}$, на обшем контуре $\Lambda$ имеем

$$
V_{\Lambda}=V_{\Lambda}^{\mathrm{el}}+V_{\Lambda}^{\mathrm{ion}}=\oint_{\Lambda} d x_{j}\left(m v_{\mathrm{el}}(x)+M v_{\mathrm{ion}}(x)\right)
$$

так что линейная комбинация циркуляций электронной и ионной скоростей сохраняется даже при наличии электромагнитного взаимодействия.

Подводя итог, можно сказать, что фазовое пространство плазмы Г в кулоновской калибровке - это пространство с координатами $\vec{A}_{\perp}(x), \vec{P}_{\perp}^{\mathrm{em}}(x) ; \vec{\xi}\left(x_{i}\right), \vec{\pi}\left(x_{i}\right) ; \vec{\Xi}\left(x_{i}\right), \vec{\Pi}\left(x_{i}\right)$ и скобками Пуассона (101) и (102). Эволюция состояния в Г определяется гамильтонианом (100).

\section{6. ЗАКЛЮЧИТЕЛЬНЫЕ ЗАМЕЧАНИЯ}

Гамильтонов подход для идеальной жидкости и плазмы, рассмотренный в настоящей работе, напоминает в некоторых аспектах гамильтонов формализм для классического поля. При таком подходе мы имеем дело с системой с бесконечным числом степеней свободы - "частицами", которые образуют сплошную среду. Эти частицы взаимодействуют с ближайшими соседями благодаря потенциальной части лагранжиана или гамильтониана подобно амплитудам в обычной теории поля. Отличие от последней, которое мы подчеркивали на протяжении статьи, заключается в том, что составляющие части жидкости изменяют свое положение в пространстве по мере эволюции, и для того чтобы иметь локальное описание жидкости в терминах $x$-зависимых переменных, нам необходимо проецирование, реализуемое, например, равенством (4). Гидродинамику можно также сравнить с механикой протяженных объектов типа $n$-бран. Эти объекты также являются $n$-мерными объектами, вложенными в пространство-время, но их составляющие неразличимы, и вследствие этого общие координатные диффеоморфизмы проявляются как калибровочная инвариантность теории. В случае жидкости предполагается, что ее составляющие различимы, и в результате только специальные диффеоморфизмы сохраняют лагранжиан, давая начало нётеровским интегралам движения.

До сих пор мы рассматривали только классическую механику жидкости и плазмы, однако, как известно, любая классическая гамильтонова система может быть подвергнута процедуре квантования. Здесь под квантованием мы подразумеваем формальную процедуру перехода от классических канонических координат к операторам, которые удовлетворяют коммутационным соотношениям, унаследованным от скобок Пуассона, и построение представления этих операторов в подходящем пространстве. Разумеется, эта процедура не имеет физического смысла в случае плазмы, но в случае жидкости было бы вполне разумно иметь теорию идеальной квантовой жидкости. Квантовая 
жидкость может быть рассмотрена как новое представление квантового поля, в котором собственное значение оператора плотности частиц может быть непрерывной функцией переменной $x$. Проблема заключается в том, что в случае обычного квантового поля нам известно только фоковское представление [15], для которого оператор плотности частиц в пространстве (или в импульсном пространстве) обладает собственными значениями, являющимися суперпозицией $\delta$-функций; это означает, что средняя плотность равна нулю, и для того чтобы рассмотреть состояния с конечной плотностью, необходимо выйти за рамки фоковского пространства.

Благодарности. Мы выражаем благодарность профессору К. Густафсону и профессору А. Разумову за плодотворные обсуждения. Помошь нашего друга, покойного профессора И. Пригожина, в период работы над этой статьей была очень важна. Работа второго автора была частично поддержана РФФИ (грант № 01-01-00201).

\section{Список литературы}

[1] Л.Д. Ландау, Е. М. Лифииц. Теоретическая физика. Т. 6. Гидродинамика. М.: Наука, 1986.

[2] F. Chen. Introduction to Plasma Physics. New York: Plenum Press, 1983.

[3] J. Marsden, A. Weinstein. Physica D. 1982. V. 4. P. 394; D. D. Holm, B. Kupershmidt, C. D. Levermore. Phys. Lett. A. 1983. V. 98. P. 389; J. Marsden, A. Weinstein. Physica D. 1983. V. 7. P. 305; D.D. Holm, J.E. Marsden, I. Ratiu, A. Weinstein. Phys. Rep. 1985. V. 123. № 1, 2. P. 1.

[4] Б. А. Дубровин, С. П. Новиков. УМН. 1989. Т. 44. № 6. С. 29; Б. А. Дубровин. Функц. анализ и его прилож. 1989. Т. 23. № 2. С. 57.

[5] A. Clebsh. J. Reine Angew. Math. 1859. V. 56. P. 1.

[6] В. Е. Захаров. ЖЭТФ. 1971. Т. 60. № 5. С. 1714.

[7] В. И. Арнольд. Математические методы классической механики. М.: Наука, 1974; V. I. Arnold, B. Khesin. Topological Methods in Hydrodynamics. Berlin: Springer, 1998.

[8] R. Jackiw. Lectures on fluid mechanics. physics/0010042.

[9] К. Ланцош. Вариационные принципы механики. М.: Мир, 1965.

[10] W. Thompson. Mathematical and Physical Papers. V.4. Cambridge: Cambridge Univ. Press, 1910.

[11] R. Salmon. Ann. Rev. Fluid Mech. 1998. V. 20. P. 225.

[12] П. А. М. Дирак. Лекции по квантовой механике. М.: Мир, 1968.

[13] Р. Балеску. Равновесная и неравновесная статистическая механика. М.: Мир, 1978.

[14] L. D. Faddeev, A. Niemi. Phys. Rev. Lett. 2000. V. 85. P. 3416; physics $/ 0003083$.

[15] С. Швебер. Введение в релятивистскую квантовую теорию поля. М.: ИЛ, 1963. 